\title{
Dancing DNA
}

\section{How Rad51 chooses the perfect partner}

Professor Hiroshi Iwasaki, Tokyo Institute of Technology aims to learn more about biological process called homologous recombination, which is crucial to maintainin whe integrity of DNA. His

means that we can learn more bout this process and a key protein called Rad51. An

increased understanding of this complex mechanism could be translated to the DNA repair processes in the human body.

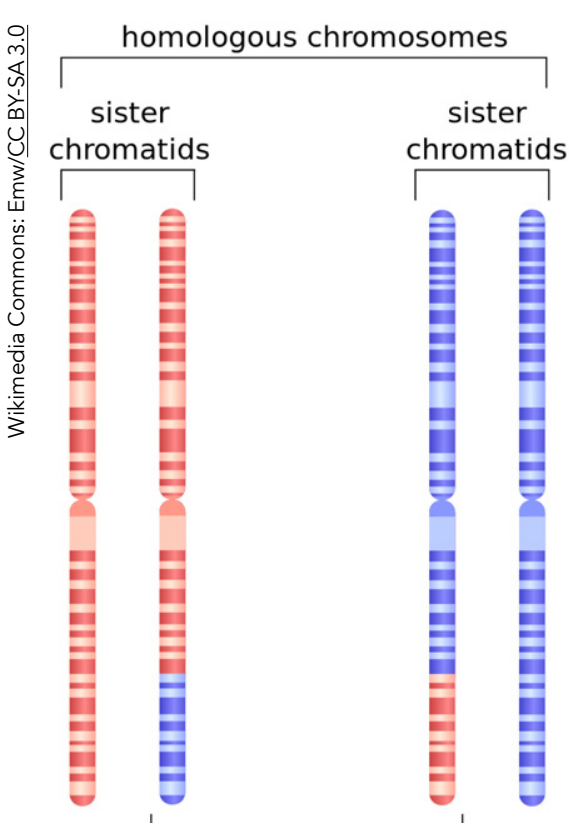

exchanged by recombination Homologous recombination is also used
during the process of meoisis which produces
reproductive cells. very day, DNA, proteins and other complex dances and rituals our bodies. They change and share the cell and the organism functioning, recombination. In meiosis (cell division it sees one donor's chromosome link arms with another and swap material to create genetic diversity; in mitosis (cell division of every other cell type). it helps preserve the integrity of genetic information.

However, this recombination and repair also behave in a more sinister manner, generating cancerous mutations and genomic rearrangements. Learning dance could help researchers understand where and how things can go wrong while highlighting potential decturg Techology Professor Hroshil was pieces together.

\section{REPAIRING DNA}

exists as two strands that are intertwined. DNA stores the genetic instructions required for the functioning growth and reproduction of all know organisms. Each strand of DNA is of building blocks that com letters $A, C, G$, and $T$. The two strands of DNA are complementary, meaning that when $A$ is present on one strand the other strand has $\mathrm{a} T$; and when $\mathrm{C}$ is present on one strand, the other strand has a G. The underlying chemistry Tand $C$ with $G$ is painng of A with strands of DNA together The order of partners, routines and stages, all to ke and to maintain genome integrity. On of these dances is called homologous the steps and actors in this convoluted these building blocks along the strands is known as the sequence of DNA. DNA molecules such as chromosomes usually contain millions of these building blocks, which are grouped into hundreds of genes, each of which is typically comprised of thousands of building blocks. Genes encode proteins, which cellular activity. The correct celch production of a fully functioning protein. When the sequence is changed, such as through the substitution of one building block for another (i.e, a mutation) - for example, an A becoming a $\mathrm{G}$ - the resultant protein is dysfunctional.

Each time a cell divides, it must first make a copy of all of its DNA (i.e., its genome) so that the new cell also carries the genetic instructions necessary for it to function. This process, known as DNA replication, is a major source of mutations since it involves copying each building block one-by-one. In addition these nutations, a more severe form (single-strand break) or both (doubletrand break) strands. When both' strands are broken, the DNA molecule is effectively separated into two meaning that a single chromosome becomes two abnormal chromosomes. Such breaks arise frequently during DNA replication, but a process known as homologous recombination is able to repair them.

But even efforts to repair such breaks can go wrong. Erroneous or unchecked homologous recombination can lead to harmful changes such as chromosomal translocations, deletion and inversions that have similar or worse consequences than the damage it was trying to fix. When mutations becumulate, and the repair of breaks
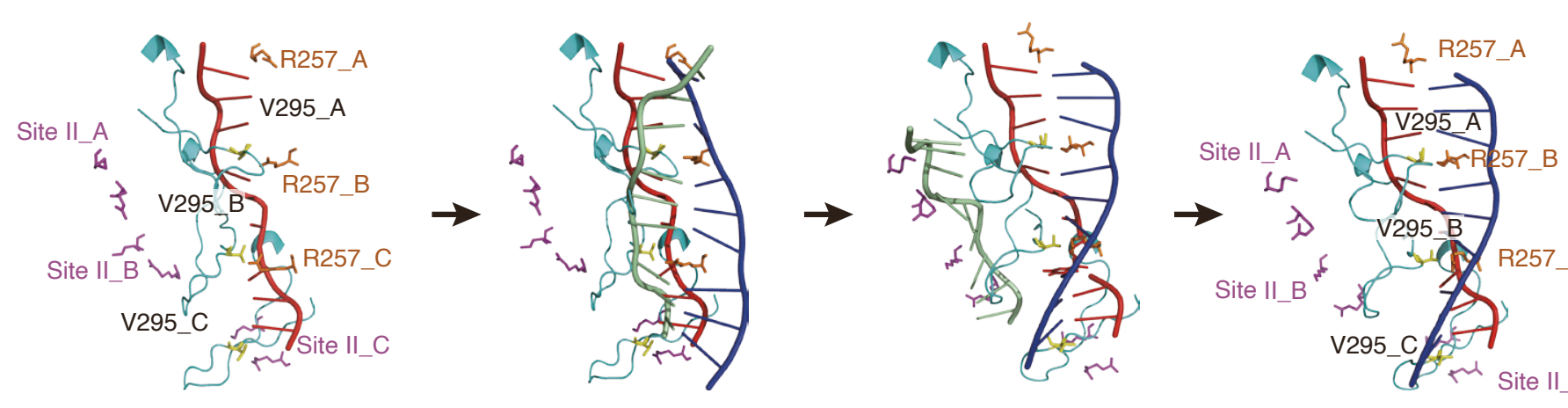

Pre-synaptic filamen

C1

C2

said to be unstable. These damaging events occur even more frequently in cells with unstable genomes, leading to further deterioration of the genetic instructions necessary for life. Such cells often become cancerous, and the eve-increasing genetic changes spur on

Why are some people more susceptible to the devastating effects of genome instability? In many cases, there are mutations in the genes involved in productive homologous recombination causing diseases such as Bloom syndrome which carry a higher risk of (amilial mutations in the BRCA1 been shown to decrease the efficiency of homologous recombination, and people whoinhert such mutated genes are at a a means there is scope to harness this repair mechanis for treatment of cancers. Early these deficiencies in homologous recombination could be exploited to stop the growth of some types of tumour Since homologous recombination is required for DNA replication, which itself is a prerequist for cell division, being able to completely inactivate homologous recombination in cancer cells may prove to be an effective strategy for halting the uncontrolled cell division associated with tumour growth.

\section{HOMOLOGOUS}

\section{RECOMBINATION 10}

cerson contains trillions of cells. In one day, a single human

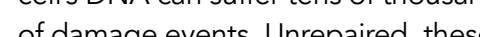

lesions can result in gene dysfunction or inaccurate gene replication, leading to cell death or - through several cell generations - gene deterioration and have an arsena of reparative tools and homologous recombination.

Homologous recombination facilitates the shuffling of genetic information with very similar (or homologous) sequences. It can be used to facilitate error-free repair of DNA double-strand breaks, alongside its role in the type

Homologous recombination facilitates the shuffling of genetic information between two different DNA molecules.

of cell division known as meiosis It is a universal mechanism, used multicellutar , bacteria, yeasts and and plant cells). protein known as 'Rad51' in humans and other eukaryotes binds single-stranded DNA (ssDNA) - generated either by a troupe of helicases and nucleases unwinding DNA at a double-strand break or a replication fork's passage over a damaged site - and searches intact double-stranded DNA (dsDNA) for sequence similarity. Once it has found a section of DNA whose sequence is highly similar to the sequence of the damaged site, the sSDNA invades the dsDNA and displaces its noncomplementary strand to create a strand is then elongted baveding eventually, cancer. Fortunately, cells between two different DNA molecules

During homologous recombination, a

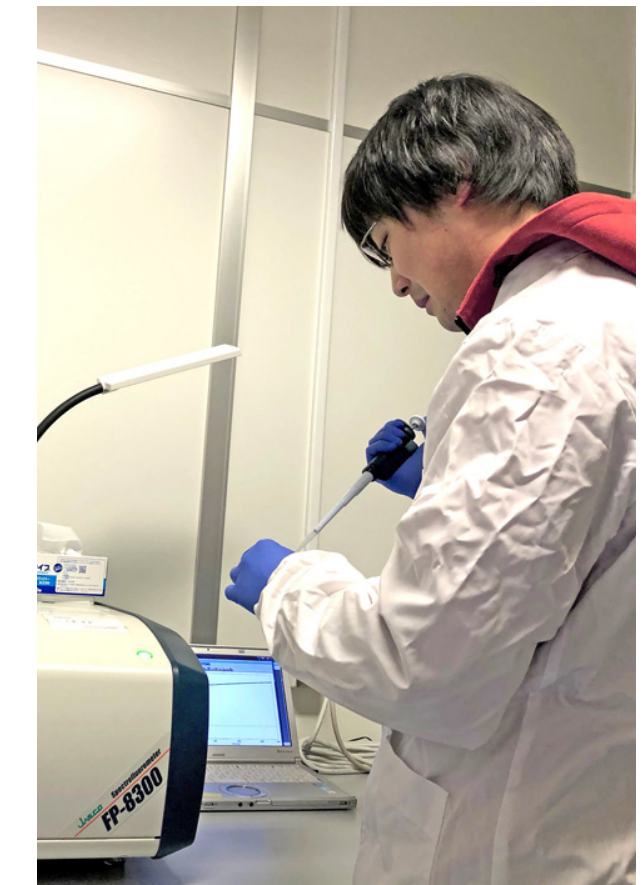

One of the team at work in the laboratory. 


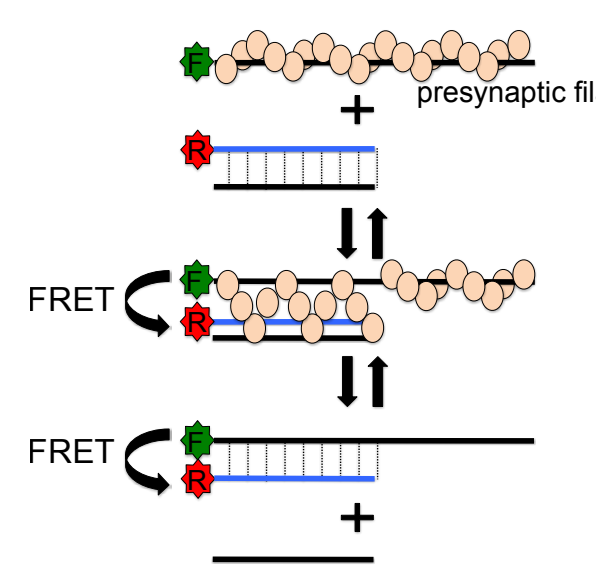

DNA Strand Pairing

Homologous recombination is a double-edged sword: it is essential for preventing genome instability but, if dysregulated, also has the potential to drastically destabilise the genome. yet fully understood. The research
of Professor Iwasaki and colleagues aims to uncover new information about the underlying mechanisms of homologous recombination.

\section{HOMOLOGOUS RECOMBINATION} CHOREOGRAPHY: A DEEP DIVE With so much riding on homologous recombination, understanding its choreography and key performers help researchers understand where and how the dance can go wrong and potentially reveal new biomarkers and anti-cancer drug targets. Knowing this, Professor Iwasaki and his team set out to study Rad51's relationship with single and double-stranded DNA and trace their steps.

\section{LEARNING FROM FRET}

Previous attempts to decipher the

molecular function of Rad51 have

focused on biochemical assays, where the Rad51 protein is purified and mixed with purified single- and doublestranded DNA in a test tube. Then, by monitoring how Rad51 binds each type the activity of Rad51. These approaches

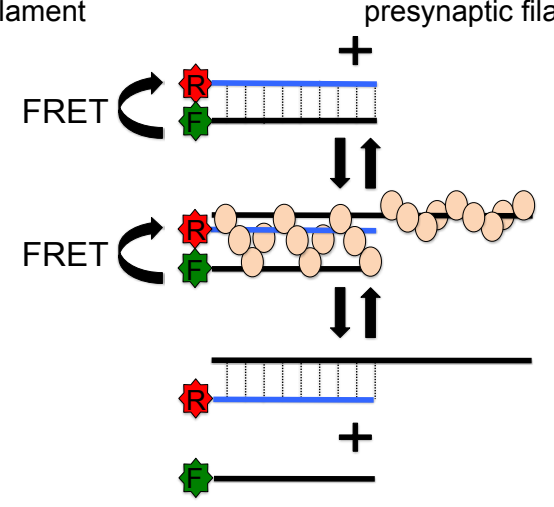

DNA Strand Displacement holding single or double-stranded DNA Site I also harbours two binding loops that the L1 and 2 loops of site $L$, along with site II. played distinct roles in the process of homologous recombination, but the differences had evaded. understanding for many years. The team employed mutant versions of yeast Rad51 to examine this process using FRET-based approaches.

Their research showed that the dance initiated by DNA breaks opens with two amino acids in site II - Arg-324 and Lys-334 - taking ssDNA by the 'hand' and forming a protein-DNA complex. An amino acid in L1 - Arg-257 - then coaxes dsDNA to the floor. Extensive homology will see L1 and L2's amino acids sequentially insert into the DNA, to displaced SDNA stable, which further assists the process.

In essence, there is a three-step model; firstly, a three-stranded DNA intermediate is formed, this intermediate released from the mature intermediate. The ability to understand more about the role of accessory factors and the regulation of Rad5T helps uncover the causes of genomic instability seen during tumour development.

\section{THE FINALE}

Professor Iwasaki's study provides and expla the kN repar process the dance of DNA strand exchange, something that has previously prove technically challenging. Expanding and enhancing the repertoire of techniques that are available to explore homologous recombination means that this research field can advance. In particular, it opens up the possibilities for studies using proteins derived from other species.

Indeed, despite being drawn with yeast proteins, homologous recombination's high conservation and similarity among organisms mean the researchers' map can be applied to humans. Further studies will be required to discover whether these findings are indeed

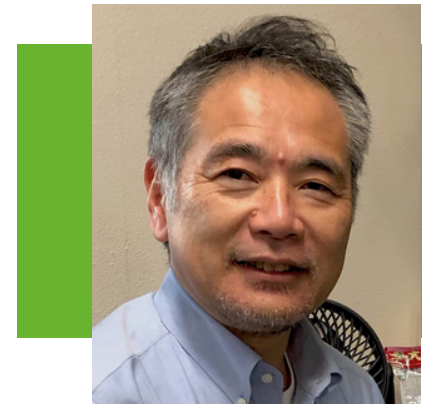

\section{Behind the Research}

\section{Professor Hiroshi Iwasaki}

E: hiwasaki@bio.titech.ac.jp T: +81459245168 W: http://www.iwasakilab.bio.titech.ac.jp then matures, and finally sSDNA is us with a comprehensive laboratory

\section{Detail} Osaka University in 1991. He has previously worked at

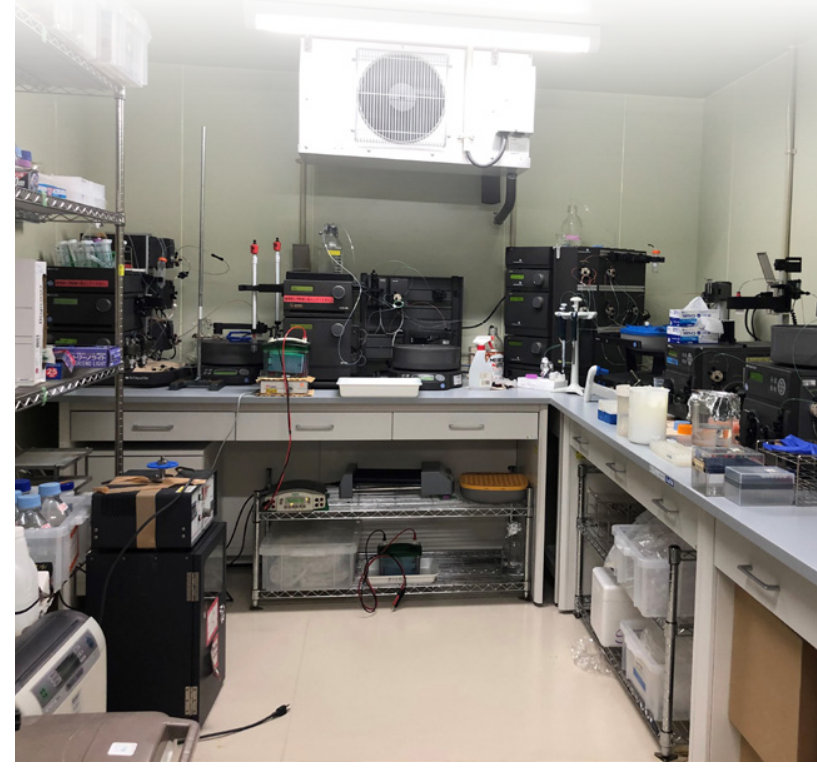
similar, whether they be from bacteria yeasts, mice, plants, or hum sites (sites and II) each resposible for
Cell Biology Center, Institute of Innovative Research

\section{Research Objectives}

Prof lwasaki examines the DNA strand exchange reaction, which is a central step of homologous recombination.

Hiroshi Iwasaki Cechnology

ori, Yokohama, Kanagawa 226-8503, Japan

Bio

Hiroshi Iwasaki obtained his PhD in Medicine from Osaka University and at Yokohama City University and is now a professor at Tokyo Institute of Technology. Prof Iwasaki's research is focused on molecular mechanisms mologous recombination and DNA repair.

\section{Funding}

JSPS (Japan Society for the Promotion of Science)

\section{Collaborators}

Kentaro Ito, Hideo Tsubouchi, Shuji Kanamaru, Bilge Argunhan, Takahisa Maki, Masayuki Takahashi

\section{References}

- Ito, K., Murayama, Y., Kurokawa, Y., Kanamaru, S., Kokabu, Y., Maki, T., et al. (2020). Real-time tracking reveals catalytic roles for the two DNA binding sites of Rad51. Nature Communications [online]. Available at: https://doi.org/10.1038/s41467-020-16750-3 - Krajewska, M., Fehrmann, R., de Vries, E. and van Vugt, M. (2015). Regulators of homologous recombination repair as novel tangets for cancer treatment. Frontiers frontiersin - Chernikova S, Game, Jand Brown, J (2012) Inhibiting homologous recombination for cancer therapy. Cancer Biology and Therapy, [online] 13(2): 61-68. Available at: https://www.ncbi.nlm.nih.gov/pmc/articles/ PMC3336066l

- Krejci, L., Altmannova, V., Spirek, M. and Zhao, X. (2012). Homologous recombination and its regulation. Nucleic Acids Research, [online] 40(13), 57955818. Available at: https://academic.oup.com/nar/ article/40/13/5795/1015492 recombination in DNA repair and DNA damage 99-113. Available at: https://www.nature.com/articles/cr20081

\section{Personal Response}

Do you think the Rad51-driven homologous repair process could be harnessed for potential

III Simple answer: yes and no. My full answer is below. homologous recombination was the best way to achieve gene therapy. But since then, we have discovered CRISPR/ CAS technology. It is my opinion, and I think most scientists would agree, hat this new technology is the most feasible method for targeted gene therapy. However interested in understanding the molecular mechanism of how Rad51 and other homologous recombination factors facilitate correct gene targeting, which is critically
important for the application of human gene therapy.

The laboratory set-up at Tokyo Institute of Technology.

| Tokyo Tech
- Li, X. and Heyer, W-D. (2008). Homologous CRISPR/CAS achieve correct targeting I am very much 